\title{
Selective Attention to Visual Stimuli Reduces Cochlear Sensitivity in Chinchillas
}

\author{
Paul H. Delano, Diego Elgueda, Carlos M. Hamame, and Luis Robles \\ Programa de Fisiología y Biofísica, Instituto de Ciencias Biomédicas, Facultad de Medicina, Universidad de Chile, Santiago 8380453, Chile
}

It is generally accepted that during periods of attention to specific stimuli there are changes in the neural activity of central auditory structures; however, it is controversial whether attention can modulate auditory responses at the cochlear level. Several studies performed in animals as well as in humans have attempted to find a modulation of cochlear responses during visual attention with contradictory results. Here, we have appraised cochlear sensitivity in behaving chinchillas by measuring, with a chronically implanted roundwindow electrode, sound-evoked auditory-nerve compound action potentials and cochlear microphonics, a measure of outer hair cell function, during selective attention to visual stimuli. Chinchillas were trained in a visual discrimination or in an auditory frequency discrimination two-choice task. We found a significant decrease of cochlear sensitivity during the period of attention to visual stimuli in the animals performing the visual discrimination task, but not in those performing the auditory task, demonstrating that this physiological effect is related to selective attention to visual stimuli rather than to an increment in arousal level. Furthermore, the magnitude of the cochlear-sensitivity reductions increased in sessions performed with shorter target-light durations $(4-0.5 \mathrm{~s})$, suggesting that this effect is stronger for higher attentional demands of the task. These results demonstrate that afferent auditory activity is modulated by selective attention as early as at sensory transduction, possibly through activation of olivocochlear efferent fibers.

Key words: auditory efferents; olivocochlear; corticofugal; attention; cochlear microphonics; top down

\section{Introduction}

Attention allows subjects to focus cognitive resources on specific stimuli while ignoring others. Although it is generally accepted that during periods of attention there are changes in neural activity in brain auditory centers (Woldorff et al., 1993; Polley et al., 2006), it is controversial whether attention can modulate auditory responses at cochlear levels (Hernandez-Peón et al., 1956; Picton et al., 1971).

Studies to assess the effect of cross-modal attention on peripheral auditory responses in animals as well as in humans have produced conflicting results. Hernandez-Peón et al. (1956) showed a reduction in click-evoked potentials recorded from the cochlear nucleus in cats while receiving stimuli of other sensory modalities. In later experiments, Oatman (1971) found an amplitude reduction of the auditory-nerve compound action potential (CAP) during periods of visual attention in cats. Similarly, in humans, the auditory-nerve component of tone-pip-evoked brainstem potentials and the amplitude of click-evoked otoacoustic emissions were significantly reduced in subjects when instructed to count letters flashed on a screen (Lukas, 1980; Puel

\footnotetext{
Received Aug. 24, 2006; revised March 9, 2007; accepted March 10, 2007.

This work was supported by Fondo Nacional de Desarrollo Científico y Tecnológico 1020970, Fundación Guillermo Puelma, Universidad de Chile Grant PG-42-2004, and PhD Scholarship Comisión Nacional de Investigación Científica y Tecnológica to P.H.D. and C.M.H. We thank Mario Ruggero for comments on a previous version of this manuscript, three unknown reviewers who helped us to improve this manuscript, and Fernando Vergara for technical assistance.

Correspondence should be addressed to Dr. Luis Robles, Programa de Fisiología y Biofísica, Instituto de Ciencias Biomédicas, Facultad de Medicina, Universidad de Chile, Casilla 70005 Santiago 7, Chile. E-mail: Irobles@med.uchile.cl.

DOI:10.1523/JNEUROSCI.3702-06.2007

Copyright $\odot 2007$ Society for Neuroscience $\quad$ 0270-6474/07/274146-08\$15.00/0
}

et al., 1988). However, other research groups failed to replicate these findings and attributed the observed decrease in cochlear responses to head or body movements (Dunlop et al., 1965; Wickelgren, 1968). In humans, paying attention to clicks during an auditory discrimination task, as opposed to reading a book and ignoring the clicks, produced no changes in click-evoked auditory nerve responses recorded from the external ear canal, despite a significant enhancement in click-evoked cortical potentials (Picton et al., 1971; Picton and Hillyard, 1974). Also, in another study, no significant changes were observed in evoked otoacoustic emissions recorded in subjects performing the same visual selective attention task used previously by Lukas (1980) and Puel et al. (1988) (Avan and Bonfils, 1992). Because of these contradictory results, the proposition that cochlear sensitivity is reduced during cross-modal attention has remained controversial.

In this study, we measured cochlear sensitivity by recording $\mathrm{CAP}$ and cochlear microphonic $(\mathrm{CM})$ potentials from the round window of chinchillas while they performed a visual or an auditory two-choice discrimination task. We demonstrated that cochlear sensitivity is transiently reduced during selective attention to visual stimuli, probably through activation of olivocochlear efferent fibers. The main technical advantages in our experimental design that could have helped the positive outcome of our experiments are as follows: (1) the use of a visual cue that delimits a short period (a few seconds) of high attentional load before target discrimination, (2) the assessment of sensitivity changes by recording auditory-nerve CAPs that report larger efferent effects than otoacoustic emissions (Puria et al., 1996), (3) the use of a round-window electrode that allowed us to measure reliable co- 

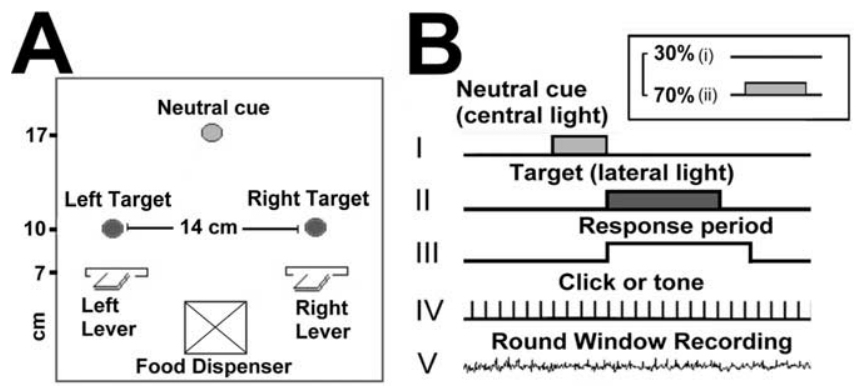

Figure 1. Visual discrimination task. $A$, Schematic diagram of the front panel of the operant cage. $\boldsymbol{B}$, Time sequence of the task. I-III, A neutral cue (central light) was turned on before one of two lateral lights (targets); the chinchilla had five seconds to respond by pressing a lever under the target light. IV, Simultaneously an irrelevant click and/or tone train was presented at 1- $4 \mathrm{~Hz}$ rate. $\mathrm{V}$, The round-window electrical signal was acquired during the task. Inset, Modified task in which only $70 \%$ of the trials began with the neutral cue.

chlear potentials with amplitudes of tens to hundreds of microvolts, in contrast with using an external-ear canal electrode that records potentials in the order of $1 \mu \mathrm{V}$ (Picton et al., 1971), and (4) sampling and averaging electrical responses with a time resolution $(500 \mathrm{~ms})$ that made it possible to detect transient modulations of cochlear potentials related to selective visual attention.

\section{Materials and Methods \\ Subjects and apparatus}

All procedures involving animals were made in accordance with the local committee of Bioethics (Comité de Bioética Animal \#098 Facultad de Medicina, Universidad de Chile) and National Institutes of Health Guidelines for the Care and Use of Laboratory Animals (publication number 86-23, revised 1996). A total of 14 chinchillas (Chinchilla laniger) weighing between 350 and $650 \mathrm{~g}$ at the start of training were used. Ten chinchillas were trained in a visual discrimination task and four in an auditory frequency discrimination task. Seven chinchillas were successfully implanted with a cochlear electrode positioned at the round window and recorded during behavior. From these chinchillas, five were recorded during the visual discrimination task and two during the auditory frequency discrimination task. All chinchillas were individually housed in temperature-controlled conditions and with an alternating dark/light cycle (lights off from 8:00 A.M. to 8:00 P.M.). They were given water ad libitum and were food deprived, maintaining $80-90 \%$ of their ad libitum-feeding weight during the experimental period. Experiments were performed in an operant mesh cage ( $32 \mathrm{~cm}$ long, $27 \mathrm{~cm}$ wide, and 29 $\mathrm{cm}$ high), that was located inside a double-walled sound-attenuating room, one meter above the room floor and $1.4 \mathrm{~m}$ from the ceiling. The front panel of the cage had three different lights (Fig. 1 A): a central light (neutral cue) above a magazine food dispenser and two lateral lights (targets) above two levers that were located seven centimeters above the cage floor. The target lights, central light and the magazine food dispenser were 10,17 , and $0.5 \mathrm{~cm}$ above the cage floor, respectively.

\section{Behavioral procedures}

Before electrode-implantation surgery, all animals were trained $5 \mathrm{~d}$ a week, over $\sim 3$ months with two daily sessions of 100 trials each. One week after surgery, chinchillas resumed their training and their cochlear potentials were recorded while performing the discrimination tasks. Recording continued until the implant anchors loosened or the quality of the electrical signals from the cochlear electrode was degraded. The operant cage, stimulus presentation and data acquisition were controlled by a custom-made computer program (developed in LabWindows/CVI environment; National Instruments, Austin, TX) and a digital interface.

Visual discrimination task. Ten chinchillas were trained in the visual discrimination task. Each trial began (Fig. $1 B$ ) with the onset of the neutral cue (central light) with a duration of $2 \mathrm{~s}$, after which, randomly, the right or left target light was turned on for a period of $4 \mathrm{~s}$. Chinchillas were trained to respond by pressing the corresponding lever. A response in the lever located below the target light made during the response period (within five seconds from the onset of the target light) was defined as a correct response and was rewarded with a pellet. Incorrect responses were defined as responses made during the response period in the lever opposite to the target light.

In both the visual and auditory discrimination tasks, correct responses were rewarded with a $45 \mathrm{mg}$ precision pellet (Noyes PJNI-0045 Chinchilla Food Pellet; Research Diets, New Brunswick, NJ), and the intertrial time interval (ITI) varied randomly between 27 and $33 \mathrm{~s}$. Incorrect responses and responses made during the ITI period were punished with a timeout period of $40 \mathrm{~s}$, during which all lights were turned off. Trials in which chinchillas did not respond to the light- or tone-target were defined as omission trials and were not punished. The behavioral variables measured in both tasks were latency of lever-pressing response (time between the onset of the light- or tone-target and the lever-pressing response, with $1 \mathrm{~ms}$ resolution), accuracy (i.e., [correct responses/(correct responses + incorrect responses)] $\times 100$ ), number of responses during the ITI, and number of omitted trials.

To compare behavioral variables at different levels of attentional demand before implantation surgery for five of the 10 chinchillas trained in the visual task, the target-light duration was progressively reduced across sessions from 4 to $0.5 \mathrm{~s}$, always maintaining an accuracy $>80 \%$. The reduction of the target-light duration from 4 to $0.5 \mathrm{~s}$ required several weeks of additional training. After electrode implantation surgery, five successfully implanted chinchillas (three from the group in which the target light was reduced before surgery and two from the group in which it was not reduced) were retrained in the visual task beginning with a target duration of $4 \mathrm{~s}$. For three of these chinchillas (the same trained previously to $0.5 \mathrm{~s}$ before surgery), the target duration was progressively shortened to $1.5 \mathrm{~s}$ in one and to $0.5 \mathrm{~s}$ in two.

Auditory frequency discrimination task. Four chinchillas were trained to discriminate between two auditory target tones of different frequencies in a task designed as a control to test whether any possible effect observed in the visual task was caused by selective visual attention or by an unspecific arousal effect. Seventy percent of the trials began with the onset of the neutral cue (central light) with a duration of $2 \mathrm{~s}$, after which randomly one of the two tones of different frequencies was delivered (see Fig. 6) (left target tone, $1200 \mathrm{~Hz}$; right target tone, $3600 \mathrm{~Hz} ; 3810 \mathrm{~ms}$ duration). Each target tone was preceded by a reference tone $(2000 \mathrm{~Hz})$ of $190 \mathrm{~ms}$ duration to facilitate the frequency discrimination. Chinchillas were trained to press the left and right levers with the 1200 and $3600 \mathrm{~Hz}$ target tones, respectively. Target and reference tones were delivered at levels $50-70 \mathrm{~dB}$ sound pressure level (SPL). Correct responses made between 300 and $5000 \mathrm{~ms}$, measured from the onset of the reference tone, were rewarded with pellets. Responses made between 0 and $300 \mathrm{~ms}$ measured from the onset of the reference tone (anticipatory) and during the ITI period, and incorrect responses made during the response period (300-5000 ms after the onset of the reference tone) were punished.

\section{Surgical procedures}

Chinchillas were anesthetized with ketamine $(20 \mathrm{mg} / \mathrm{kg}$, i.m.) and acepromazine $(0.7 \mathrm{mg} / \mathrm{kg}, \mathrm{i} . \mathrm{m}$.). The anesthetic protocol included atropine sulfate $(0.04 \mathrm{mg} / \mathrm{kg}$, i.m.). Supplemental doses of ketamine $(10 \mathrm{mg} /$ $\mathrm{kg}$ ) were given at $\sim 30-45 \mathrm{~min}$ intervals, depending on the anesthetic level judged by the foot-withdrawal reflex. Body temperature was maintained at $36.5^{\circ} \mathrm{C}$. The auditory bulla was opened and a low-impedance nichrome electrode $(100 \mu \mathrm{m})$ was positioned in the round window of the cochlea. The electrode was fixed to the skull with dental acrylic cement. Three screws, 1/16 inch, (Small Parts, Miami Lakes, FL) were implanted on the cranium. One of them was used as a differential electrode and another as ground. The round-window electrode and two wires connected to the skull screws were attached to a three-pin connector, which was fixed to the skull with dental acrylic cement, and permitted the recording of cochlear potentials in the behaving animals. Chinchillas were allowed to recover for at least 1 week after implantation surgery before beginning with the awake recordings. A steel tube was implanted in the bulla of one of the chinchillas (trained in the visual discrimination task) that allowed us to measure sound pressures in the bulla simultaneously to cochlear potentials. At the end of experiments, deeply anes- 
Table 1. Behavioral performance of chinchillas trained in the visual and auditory discrimination tasks

\begin{tabular}{|c|c|c|c|c|}
\hline & Accuracy (\%) & Mean response latency (ms) & Omissions (n/100 trials) & ITI responses (n/100 trials) \\
\hline \multicolumn{5}{|c|}{ Behavior summary before implantation surgery } \\
\hline \multicolumn{5}{|c|}{ Visual task } \\
\hline Target light $4.0 \mathrm{~s}(n=10)$ & $94.0 \pm 3.3$ & $2024 \pm 366$ & $38.2 \pm 24.0$ & $4.9 \pm 3.9$ \\
\hline Target light $0.5 \mathrm{~s}(n=5)$ & $89.4 \pm 3.9^{*}$ & $1418 \pm 292^{* *}$ & $13.2 \pm 2.5^{* *}$ & $6.7 \pm 4.1$ \\
\hline \multicolumn{5}{|l|}{ Auditory task } \\
\hline Target tone $4.0 \mathrm{~s}(n=4)$ & $89.7 \pm 7.5$ & $2439 \pm 168$ & $16.5 \pm 6.1$ & $7.2 \pm 5.4$ \\
\hline \multicolumn{5}{|c|}{ Behavior summary during recordings sessions } \\
\hline \multicolumn{5}{|c|}{ Visual task } \\
\hline Target light $4.0 \mathrm{~s}(n=5)$ & $88.1 \pm 9.5$ & $2324 \pm 413$ & $42.3 \pm 15.2$ & $5.7 \pm 6.4$ \\
\hline Target light $0.5 \mathrm{~s}(n=2)$ & $80.5 \pm 6.2$ & $1786 \pm 156$ & $18.4 \pm 16.6$ & $3.2 \pm 2.1$ \\
\hline \multicolumn{5}{|l|}{ Auditory task } \\
\hline Target tone $4.0 \mathrm{~s}(n=2)$ & $77.7 \pm 2.3$ & $2421 \pm 634$ & $22.1 \pm 0.4$ & $5.4 \pm 3.3$ \\
\hline
\end{tabular}

Data are shown as mean \pm SD. Statistical comparisons were calculated with unpaired $t$ test for behavioral variables acquired in the two target-light duration conditions before implantation surgery ${ }^{*} p<0.05 ;{ }^{* *} p<0.01$.

thetized chinchillas were humanely killed with sodium thiopental (200 $\mathrm{mg} / \mathrm{kg}$, i.c.).

\section{Physiological procedures}

Auditory stimuli to assess CAP and CM responses. In the seven implanted chinchillas, the sensitivity of the cochlea was appraised by the amplitude of the CAP measured at the round window in response to a train of alternating-polarity clicks. In three of these implanted animals (two in the visual task and one in the auditory task), tone-evoked CM potentials were measured in addition to the click-evoked CAPs by using a compound stimulus consisting of clicks of alternating polarity followed within a $40 \mathrm{~ms}$ period by tone bursts. Clicks (100 $\mu$ s wide) and tone bursts at frequencies of $1,2,4,6$, and $8 \mathrm{kHz}$ in the visual task and $1200 \mathrm{~Hz}$ in the auditory task were digitally generated at $100 \mathrm{kHz}$ with a National Instruments Board (PC-MIO-16E4). Tone bursts had a $5 \mathrm{~ms}$ ramp and a total duration of 100-200 ms. Click and tone trains were delivered through a loudspeaker (XS-302F; Sony, Tokyo, Japan; $100 \mathrm{~W}$, frequency response $20-20,000 \mathrm{~Hz}$ ) located $120 \mathrm{~cm}$ above the floor of the operant cage. Auditory stimuli were delivered at presentation rates between 1 and $4 \mathrm{~Hz}$ and levels at 50-70 dB SPL. We performed sound pressure calibrations inside the operant cage with a 0.5 inch Bruel and Kjaer microphone. The sound pressure level inside the bulla of the chinchilla implanted with a steel tube was measured using a small microphone (BL-1785; Knowles Electronics, Itasca, IL).

Data acquisition and analysis. The electrode signal was amplified $10,000 \times$, filtered $(100 \mathrm{~Hz}-10 \mathrm{kHz})$ and digitized at $20 \mathrm{kHz}$ with a PCI6024-E National Instruments board housed in a Pentium IV personal computer. We stored $10-15 \mathrm{~s}$ of data in each trial, always triggered $2 \mathrm{~s}$ before the onset of the neutral cue. All data were analyzed off-line by custom-made software written in C language (LabWindows/CVI from National Instruments). Recording trials with motor artifacts were eliminated using an amplitude threshold criterion. Compound action potential amplitudes were calculated from the peak-to-peak signal in microvolts. The magnitude of cochlear microphonic potentials was computed with the power spectrum of the fast-Fourier transform.

We performed statistical comparisons mainly between cochlear potentials recorded during correct and omitted trials. Chinchillas presented high percentages of omission in both tasks, despite maintaining high accuracies of target discrimination (Table 1). Because of this characteristic of chinchillas' behavior, most of the trials in the task were correct or omitted, whereas few trials were incorrect and even fewer trials had ITI responses. We have assumed that there is a plausible difference between the attentional state of chinchillas during correct trials compared with omission trials. In contrast, we could not assume that chinchillas were less attentive during incorrect than during correct trials. For these reasons, the mean amplitudes of the CAP and CM for correct trials were compared with those for omission trials. The measurements acquired during the period from the onset of the neutral cue up to the mean latency of responses were compared using unpaired $t$ test analysis. Values measured after the animal responses were not analyzed because they could be altered by pellet-dispenser noise, animal movements, or arti- facts produced by chewing. To determine the relation between length of target light (attentional load of the task) and magnitude of CAP reduction, we used the Spearman correlation analysis $(\alpha=0.05)$.

To compare the changes observed in CAP and CM potentials, we calculated the changes in amplitude of the cochlear potentials (CM or $\mathrm{CAP}$ ) in decibels, using as reference the average of the three mean amplitudes obtained in the two seconds previous to the onset of the neutral cue with the following equation: $\mathrm{dB}$ (change) $=20^{\star} \log _{10}$ [amplitude of cochlear potential/mean reference potential].

\section{Results}

We found a significant reduction in CAP amplitude within the period of selective attention to visual stimuli in the five implanted chinchillas studied in the visual discrimination task (Table 2). Figure $2 \mathrm{~A}$ displays mean CAP amplitudes measured in one animal for correct-response trials (solid squares) and omission trials (open circles), during a period starting before visual stimuli presentation and extending well after the motor responses. However, as mentioned above, the results were always analyzed only for measurements obtained during the period up to the mean latency of responses, to avoid any possible artifacts caused by animal movements, pellet-dispenser noise, or chewing noise. A progressive decrease in CAP amplitude, in the correct-response trials, but not in the omission trials, was consistently observed in the five chinchillas trained in the visual discrimination task. As shown in Figures 2-5, the CAP reduction started during the period of presentation of the neutral cue and continued during the target-light period.

In an attempt to increase the attentional demands of the visual discrimination task, in five of the chinchillas the duration of the target light was progressively reduced along days from 4 to $0.5 \mathrm{~s}$ (Muir et al., 1994; Chiba et al., 1999). The effect of reducing the light duration in the chinchillas trained before implantation surgery was a significant decrease in accuracy and mean response latency (Table 1). The same reduction in target-light duration in two of the five implanted animals during the recording sessions produced a similar, but not significant, decrease in accuracy and mean response latency (Table 1 ). As shown in Figure $2 B$, decreasing the target-light duration during the recording sessions produced progressively greater CAP reductions. In the two animals tested, there was a significant correlation between the magnitude of the CAP reduction and the duration of the target light, as illustrated in Figure $2 C(p<0.01$, Spearman test).

To test whether CM increases accompanied the CAP reductions (as shown previously for electrical stimulation of cochlear efferents) (Guinan, 1996) (see Discussion) in two animals trained in the visual discrimination task, we measured almost simulta- 
Table 2. Summary of recording sessions in chinchillas performing the visual and auditory discrimination tasks

\begin{tabular}{|c|c|c|c|c|c|c|}
\hline & $\begin{array}{l}\text { Total c } \\
\text { sessio }\end{array}$ & ording & Percentage of sessions with reduction of & & & Shortest target duration used during \\
\hline & days & sessions $^{a}$ & cochlear sensitivity $(\%)^{b}$ & CAP reduction ${ }^{c}(\mathrm{~dB})$ & CM increase ${ }^{c}(d B)$ & awake recordings (s) \\
\hline vis_Ch1 & 18 & $18(43)$ & 94.4 & $1.9(3.3)$ & $2.6(4.8)$ & 0.5 \\
\hline vis_Ch2 & 27 & $32(54)$ & 100.0 & $2.1(3.6)$ & $3.5(5.9)$ & 0.5 \\
\hline vis_Ch3 & 7 & $7(16)$ & 85.7 & $1.1(2.1)$ & $\mathrm{nt}$ & 4.0 \\
\hline vis_Ch4 & 6 & $4(8)$ & 100.0 & $1.8(3.8)$ & nt & 4.0 \\
\hline vis_Ch5 & 25 & $6(49)$ & 83.3 & $0.6(0.9)$ & $\mathrm{nt}$ & 1.5 \\
\hline aud_Ch6 & 17 & $11(34)$ & 0.0 & $\mathrm{nc}$ & nc & 4.0 \\
\hline aud_Ch7 & 7 & $9(15)$ & 0.0 & $\mathrm{nc}$ & $\mathrm{nt}$ & 4.0 \\
\hline
\end{tabular}

The first five rows show data from chinchillas (Ch) trained in the visual task (vis_Ch1 to vis_Ch5) and the last two rows from those trained in the auditory task (aud_Ch6 and aud_Ch7). nt, Not tested; $\mathrm{nc}$, no change.

${ }^{a}$ Number of recording sessions with at least 20 correct responses, which were used for cochlear potential analysis. In parentheses is the total number of sessions in each animal.

${ }^{b}$ The percentage of recordings with reduction of cochlear sensitivity was calculated from the recordings with at least 20 correct responses that had CAP reduction and CM increase (for vis_Ch1 and vis_Ch2) or only CAP reduction (for vis_Ch3, vis_Ch_4, and vis_Ch5).

'Mean and maximum amplitude changes (in parentheses) measured in each chinchilla (in decibels).

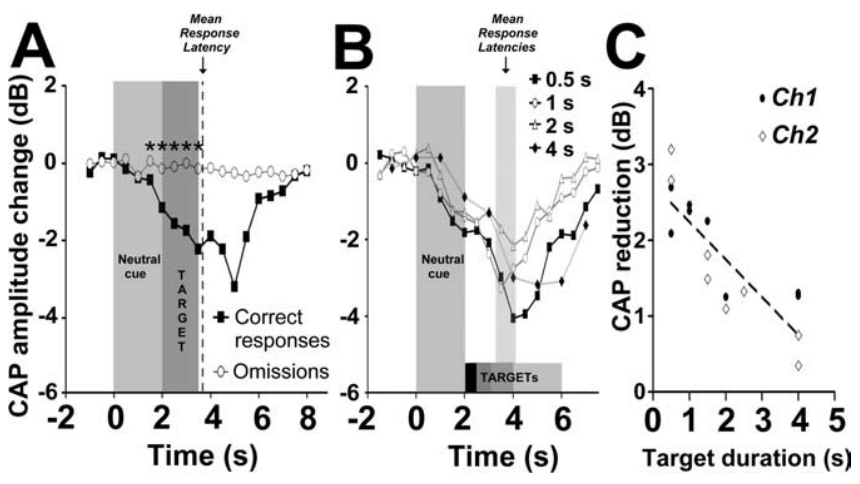

Figure 2. CAP results. $A$, Example of CAP reductions in correct-response trials (solid squares) compared with omission trials (open circles) during the period of visual attention (in this and the following figures; light gray, neutral-cue period; dark-gray, target-light period). Symbols on each trace represent CAP amplitude changes measured in decibels, referenced to the average amplitude of the potentials measured before the onset of the neutral cue. We show CAPs from a single recording session ( 100 trials) in response to a $2 \mathrm{~Hz}$ click train. Significant CAP reductions, calculated for pairs of values measured from the neutral-cue onset up to the mean response latency, are indicated by asterisks (unpaired $t$ test; $p<0.05$ ). Accuracy was $95 \%$ and mean response latency, always measured from the target-light onset, was $1688 \mathrm{~ms}$ (vertical segmented line). $\boldsymbol{B}$, Progressively greater (AP reductions for gradual decrease of target-light duration (4- $0.5 \mathrm{~s}$ ) in one of two tested animals showing correlation between increase in attentional demands of the task and amount of CAP reduction. The curves shown in this panel for the different target durations correspond to the first recording session of 100 trials (of two daily sessions). The gray bar (arrow) indicates the interval (1274-2109 ms) of mean correctresponse latencies measured from the target-light onset in the $4 \mathrm{~d}$. C, Correlation between target duration and CAP reduction. We show data from two chinchillas (solid and open symbols) in which we successfully reduced the target duration from $4 \mathrm{~s}$ to $0.5 \mathrm{~s}$ during successive recording sessions. We found a significant correlation between target duration and CAP reduction $(p<0.01$, Spearman test). The equation of the linear regression shown in this figure is $y$ $(\mathrm{dB})=-0.5 \times(\mathrm{s})+2.75 ;\left(R^{2}=0.73\right)$. In $\boldsymbol{B}$ and $\boldsymbol{C}$, only sessions in which chinchillas had accuracies $>75 \%$ and made at least 20 correct responses are shown.

neously both CAP and CM while the animals performed the visual discrimination task. The use of a compound stimulus, consisting of a click followed by a tone burst, allowed us to measure CAP and CM within a $40 \mathrm{~ms}$ interval. We found a significant augmentation of CM amplitude concomitant to the reduction in CAP amplitude during the period of selective attention to visual stimuli in the two animals tested. Figure $3 A$ displays the increase in CM amplitudes (solid squares) accompanying the CAP amplitude reductions (open circles) for correct-response trials measured during the period of visual stimuli presentation. The inset in this figure displays examples of CAP reduction and CM increment that compare the averaged waveforms of the two cochlear
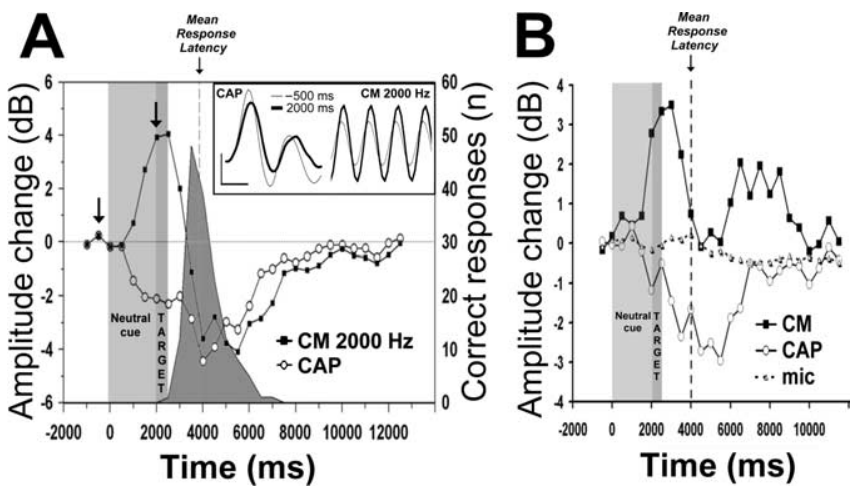

Figure 3. Results for compound stimuli. $A$, Example of $C M$ augmentation (solid squares) concomitant to CAP reductions (open circles) during the period of visual attention. Symbols on each trace represent CAP and CM amplitude changes measured in decibels, referenced to the average amplitude of the respective cochlear potential measured before the onset of the neutral cue. The figure also shows a histogram for the latencies of correct responses to the target (dark-gray shaded area; scale in right $y$-axes). The top right inset shows examples of averaged CAP and CM traces from $-500 \mathrm{~ms}$ (thin trace) and $2000 \mathrm{~ms}$ (thick trace; see vertical arrows in $A$; calibration: $0.5 \mathrm{~ms}, 40 \mu \mathrm{V}$ for $(\mathrm{AP}, 80 \mu \mathrm{V}$ for $(\mathrm{CM})$. In this case, accuracy was $80.2 \%$ and mean response latency measured from the target-light onset was $1833 \mathrm{~ms}$ (vertical segmented line). $B$, Control of sound pressure level within the chinchilla's bulla using a microphone. Changes of sound pressure (triangles), CAP decreases (open circles), and CM augmentations (solid squares) during the period of visual attention (symbols on each trace represent changes measured in decibels, as in A). Notice that neither (AP decreases nor CM increases are caused by changes in sound pressure level reaching the cochlea. Accuracy was $85.3 \%$ and mean response latency measured from the target-light onset was $2034 \mathrm{~ms}$ (vertical segmented line).

potentials sampled at two different time points of the behavioral task (see arrows at -500 and $2000 \mathrm{~ms}$ ). Figure $3 \mathrm{~A}$ also displays a histogram of the latencies of correct responses to targets (dark gray shaded area), which shows that motor responses begin $\sim 500$ ms after the offset of the target light. Therefore, the reduction in cochlear sensitivity (CM increase and CAP reduction) is produced well before the motor response and could not be caused by it. However, the sharp negative slopes in the amplitude changes present in both cochlear potentials after the target-light offset are probably related to the motor responses.

To discard the possibility that the modulations in cochlear responses during periods of visual attention were caused by changes in stimulus intensity attributable to head movements, in one animal we measured the sound pressure within the bulla (through an implanted steel tube). As illustrated in Figure $3 B$, CAP reductions (open circles) and CM augmentations (solid squares) were observed without significant changes in the sound 


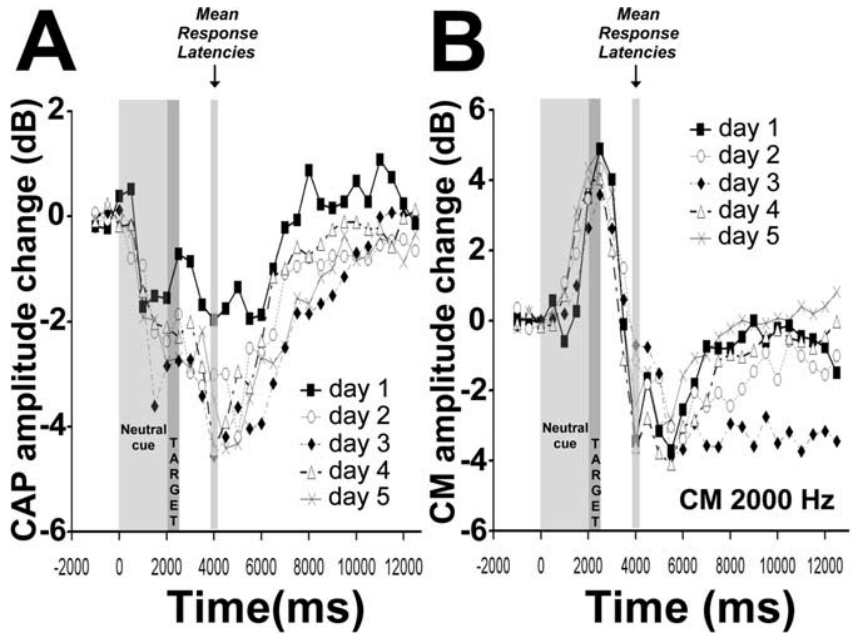

Figure 4. Reliability of the effect in cochlear potentials. $A, B$, Examples of CAP reductions $(\boldsymbol{A})$ and $C M$ augmentations $(\boldsymbol{B})$ recorded in one chinchilla during 5 consecutive days in identical stimulus conditions. In all cases, compound stimuli were delivered at $2 \mathrm{~Hz}$ rate and accuracy was $>75 \%$. The gray bars with arrows indicate the interval (1907-2091 ms) of mean correctresponse latencies measured from the target-light onset in the $5 \mathrm{~d}$. Symbols on each trace represent cochlear potentials amplitude changes measured in decibels, referenced to the average amplitude of the respective cochlear potential measured before the onset of the neutral cue.

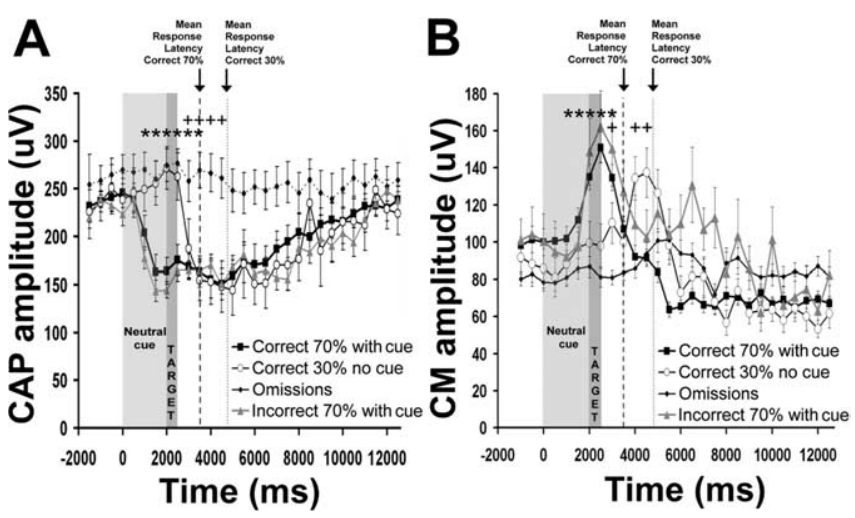

Figure 5. Results for the modified $70 / 30$ visual task. $\boldsymbol{A}, \boldsymbol{B}, \mathrm{CAP}$ reductions $(\boldsymbol{A})$ and $C M$ augmentations $(\boldsymbol{B})$ in the modified task (solid squares, correct responses in the $70 \%$ of trials with the neutral cue; open circles, correct responses in the $30 \%$ without the neutral cue; triangles, incorrect responses in the $70 \%$ of the trials with the neutral cue; solid diamonds, omission trials). Significant CAP reductions and CM increases in correct trials compared with omission trials are indicated by asterisks and by crosses for trials with and without the neutral cue, respectively (unpaired $t$ test, $p<0.05$ ). Notice that incorrect trials with the neutral cue also present CAP reductions and CM increases similar to those in the correct trials with the neutral cue. Mean correct-response latencies measured from the target-light onset, in trials with and without the neutral cue are shown with segmented lines ( $1520 \mathrm{~ms}$, cued trials; $2832 \mathrm{~ms}$, no cue trials). The mean incorrect-response latency was $1103 \mathrm{~ms}$.

pressure measured simultaneously inside the bulla by the microphone (triangles).

The significant modulations in cochlear potentials observed during the periods of visual attention amounted up to $4 \mathrm{~dB}$ for CAP reductions and $6 \mathrm{~dB}$ for $\mathrm{CM}$ increases (Table 2), and are within the range of effects produced by electrical activation of olivocochlear fibers in anesthetized animals (Galambos, 1956; Gifford and Guinan, 1987). These modulations in both cochlear potentials were extremely reliable across sessions. Figure 4 illustrates the consistency of the CAP reductions and CM increases observed in one animal throughout sessions performed during five consecutive days, under identical stimulus conditions. Furthermore, as depicted in Table 2, the reductions in cochlear sensitivity during periods of visual attention were found in all animals and in $83-100 \%$ of the sessions, with at least 20 correct responses recorded in each animal.

To evaluate the temporal relation between the modulation of cochlear potentials and the beginning of the attentional process, we modified the visual discrimination task to another in which the neutral cue was presented only in $70 \%$ of the trials, whereas in the other $30 \%$, the target light was presented without the neutral cue (Fig. $1 B$, inset). Using this protocol, correct trials without the neutral cue exhibited CAP amplitude reductions (Fig. 5A) and $\mathrm{CM}$ amplitude increases (Fig. $5 B$ ) similar to those observed in the correct trials with the neutral cue, but delayed by a period comparable with the duration of the neutral cue, thus showing that these effects are related to the beginning of the attentional process. The cochlear recordings depicted in Figure 5 are from an animal in which there were enough incorrect responses to be analyzed. In this case, the CAP reductions and CM increases recorded in the incorrect trials with the neutral cue were similar to those obtained in the correct trials with the neutral cue.

Finally, we trained four chinchillas in an auditory frequencydiscrimination two-choice task, designed as a control to demonstrate that the attentional effect observed in the visual task was caused by selective attention to visual stimuli and not by an unspecific arousal effect produced by the presence of the neutral cue. In this auditory discrimination task, an auditory target was preceded by the same visual neutral cue that we used in the visual discrimination task (Fig. 6A). The presence of the neutral cue reduced the mean response latency to correct responses (Fig. $6 B$ ), as it did in the visual task (Fig. 5) (Hamame et al., 2006). However, in the two animals implanted with the round-window electrode and tested in the auditory task, there were no changes in the amplitude of cochlear potentials during the presentation of the neutral cue in contrast to the visual task (Fig. 6C).

\section{Discussion}

In the present study, we demonstrate that during selective attention to visual stimuli in a visual discrimination task there are CAP reductions and $\mathrm{CM}$ increments that reflect a transient decrease in cochlear sensitivity, probably produced through activation of olivocochlear efferent fibers. We also show that the magnitude of the CAP reduction is dependent on the length of the visual target.

\section{Reduction of cochlear sensitivity during visual selective attention}

After the controversial work of Hernandez-Peón et al. (1956), in better-controlled experiments, Oatman reported CAP reductions in cats attending to visual stimuli, but without significant changes in CM (Oatman, 1971, 1976; Oatman and Anderson, 1977). We found a consistent decrease in CAP amplitude in the period of increased visual attention during the discrimination task, thus confirming Oatman's results. However, we have extended his findings by recording the temporal course of the CAP reduction during the visual attention period and by demonstrating a concomitant increase of CM during visual attention. It is likely that Oatman's failure to observe significant CM increases during visual attention was attributable to the limited time resolution of the recording methods available at that time. Although $\mathrm{CM}$ increases have been indirectly suggested in chronically deefferented awake chinchillas (Zheng et al., 2000), this is the first study demonstrating that CM increases and CAP reductions are present together in the behaving animal. 


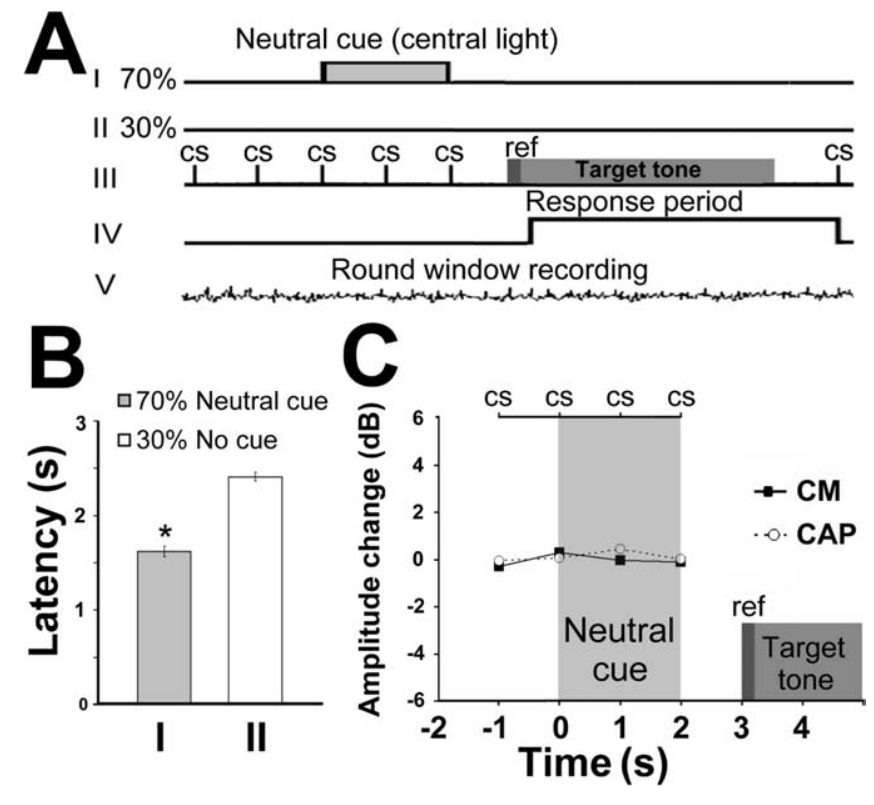

Figure 6. Auditory frequency discrimination two-choice task. $A$, Timeline of the auditory task. I, II, In $70 \%$ of the trials (I), the neutral cue was presented before the onset of a target tone, whereas (II) in the other $30 \%$ of the trials the target tone was presented without the neutral cue. III, Auditory stimuli. The compound stimuli (cs; a click of alternating polarity followed after 40 ms by a $1200 \mathrm{~Hz}$ tone) were presented at a rate of $1 \mathrm{~Hz}$. The reference tone $(2000 \mathrm{~Hz}, 190 \mathrm{~ms}$ ) was presented before one of the target tones (tone for pressing left lever, $1200 \mathrm{~Hz}$; right lever, $3600 \mathrm{~Hz}$; duration, $3810 \mathrm{~ms}$ ). IV, Chinchillas had a response window of 300-5000 ms from the onset of the reference tone. $V$, Simultaneously, we recorded the round window signal during behavior. $\boldsymbol{B}$, Mean response latency to the target tone measured from the reference tone onset, with and without neutral cue. The mean response latencies were shorter for the $70 \%$ of the trials in which the neutral cue was presented (with neutral cue, 1619 ms; without neutral cue, 2409 ms; unpaired $t$ test, $p<0.01$ ). C, (AP and CM amplitude changes (in decibels) for correct-trial responses, referenced to the mean values measured previous to the onset of the neutral cue. There were no changes in CM and CAP amplitudes during the presentation of the neutral cue when the targets were tones.

A contraction of middle-ear muscles related to body movements has been proposed as a mechanism that could account for the reductions in CAP amplitude during the period of visual attention (Starr, 1964; Carmel and Starr, 1964). However, Oatman (1971) sectioned the tendons of middle ear muscles in cats and found that the reduction in CAP amplitude was not affected. In our case, the presence of CM increments that consistently accompanied CAP reductions also makes our data incompatible with a middle-ear effect. Consequently, it appears that the reduction in cochlear sensitivity, during the period of visual attention, is not caused by middle-ear muscle contraction.

In the few sessions in which there were enough incorrect trials for analysis, these trials presented significant reductions in cochlear sensitivity during visual attention, indistinguishable to the reductions observed for correct trials (Fig. 5). This implies that chinchillas were also attentive during incorrect trials, and that the observed cochlear modulations do not depend on the cortical network involved in behavioral decisions (Romo and Salinas, 2003).

\section{Possible mechanisms of the observed cochlear-sensitivity modulation}

Auditory efferents are most probably involved in this crossmodal cochlear effect. The mammalian cochlea has two types of sensory cells, inner (IHCs) and outer hair cells (OHCs). Most afferent axons innervate IHCs, which constitute the main co- chlear sensory receptors. In contrast, OHCs receive predominantly efferent fibers originating near the medial superior olivary nuclei (Guinan, 1996; Azeredo et al., 1999), and in addition to their function as receptors they also act as mechanical effectors boosting cochlear vibrations for low-level stimuli (Dallos, 1992). Neuroanatomical evidences show that descending pathways originate in the auditory cortex and reach the cochlea via olivocochlear efferents (Warr, 1975; Feliciano et al., 1995; Mulders and Robertson, 2000). Electrical stimulation of these olivocochlear efferents in anesthetized cats decreases the amplitude of CAP (Galambos, 1956) and increases the amplitude of CM (Fex, 1959), reflecting a reduction of cochlear sensitivity (supplemental note, available at www.jneurosci.org as supplemental material). We propose that during visual attention, through corticofugal pathways, there is activation of medial olivocochlear fibers that hyperpolarizes OHCs (Oliver et al., 2000), increasing their transduction potentials, which are the principal source of CM potentials recorded at the round window (Dallos and Cheatham, 1976). The activation of the medial efferent fibers also reduces the gain of the cochlear amplifier, decreasing CAP amplitudes. However, a simultaneous activation of lateral olivocochlear efferent fibers that modulate auditory nerve responses, but not hair-cell activity, cannot be ruled out (Groff and Liberman, 2003).

\section{Temporal course of the efferent-effect and visual attention}

The temporal course of the reduction of cochlear sensitivity during visual attention was relatively slow, and probably reflects the time course of the cognitive processes rather than that of auditory efferent activation. The reductions in cochlear sensitivity that we found during the periods of attention to visual stimuli have latencies of $\sim 1 \mathrm{~s}$, measured from the onset of the neutral cue, and a time constant of 1-1.5 s. Electrical activation of olivocochlear fibers in anesthetized animals reduces CAP amplitudes with a time constant of $\sim 160$ ms (Galambos, 1956) and cochlear mechanical responses with a time constant of 10-100 ms (Cooper and Guinan, 2003). Recently, James et al. (2005) found that contralateral noise-stimulation of olivocochlear fibers produces suppression of otoacoustic emissions with a latency of $\sim 26 \mathrm{~ms}$ in chinchillas and $45 \mathrm{~ms}$ in humans. However, studies in humans have shown that the latency of attention effects, reflected by cortical potentials recorded during a cued-visual task, is $\sim 500-600$ ms (Muller et al., 1998) and that the temporal course of visual attention is also in the order of hundreds of milliseconds (Duncan et al., 1994; Ward et al., 1996). According to these data, the long latencies of the observed reductions in cochlear sensitivity most likely reflect the time course of the attentional process rather than that of efferent fiber activation. These long latencies could also explain the fact that in the modified 70/30 experiment (Fig. 5), in the no-cue condition, the amplitude changes in cochlear potentials did not occur during the target light presentations, but after their off-set.

\section{Auditory frequency-specific efferent effects}

As a control to the visual task used to measure the cross-modal effect of selective attention, we tried to find an auditory task of similar difficulty. Despite using target tones $>1.5$ octaves apart and a reference tone before the target tones (to facilitate frequency discrimination), the auditory task was more difficult to learn for chinchillas than the visual one, and the accuracy in it was lower than in the visual task (Table 1).

We did not find an efferent effect of attention in the auditory task, demonstrating that the effect observed in the visual task was attributable to selective attention to visual stimuli and not to an 
unspecific arousal effect caused by the presence of the neutral cue. However, it is possible that a frequency-specific efferent effect could be found in an auditory task specifically designed for that purpose. There is experimental evidence in anesthetized animals of a frequency-dependent loss of sensitivity in basilar membrane vibrations during efferent stimulation (Murugasu and Russell, 1996; Dolan et al., 1997) that could account for similar neural effects (Robles and Ruggero, 2001). Likewise, in a detection task in which human subjects are led to expect target tones of a particular frequency, there are frequency-specific attenuation effects in the detection of unexpected frequencies in noise (Greenberg and Larkin, 1968). Furthermore, humans in whom the auditory efferent pathway was cut, present higher detection levels of unexpected frequencies in this task, than before surgery, suggesting that the efferent system may be required to maintain an attentional bandpass filter (Scharf et al., 1997). Using a similar task, Dai et al. (1991) have shown that there is $\sim 7 \mathrm{~dB}$ of attenuation in detectability of the unexpected tones compared with the expected tone, suggestive in humans of an efferent attenuation of unattended tones of $\sim 7 \mathrm{~dB}$. This value, although similar to the reduction of cochlear sensitivity (up to $6 \mathrm{~dB}$ ) that we observed during visual attention, probably is not a measure of the same efferent effect, because we studied a cross-modal physiological effect whereas these authors measured a psychophysical intramodal frequency-specific effect (Dai et al., 1991).

Some of the proposed functions of the efferent auditory system are (1) antimasking effects in presence of noise (Kawase and Liberman, 1993), (2) protection from loud sound damage (Maison and Liberman, 2000), (3) modulation of auditory activity during awake/sleep states (Velluti et al., 1989), and (4) modulation of cochlear sensitivity during cross-modal attention (Oatman, 1971). Here, we provide clear evidence for the latter function by demonstrating a clear and repeatable reduction of cochlear sensitivity during the period of visual selective attention in animals performing a visual discrimination task. We also show that the magnitude of the cochlear sensitivity decrease depends on the duration of the target light, suggesting that this effect increases with higher attentional demands of the task. Although a direct confirmation of the involvement of auditory efferents in this effect is still lacking, the occurrence of concomitant decreases in CAP amplitude and increases in CM amplitude during the period of visual attention strongly suggests that this modulation is mediated through activation of olivocochlear efferent fibers.

\section{References}

Avan P, Bonfils P (1992) Analysis of possible interactions of an attentional task with cochlear micromechanics. Hear Res 57:269-275.

Azeredo WJ, Kliment ML, Morley BJ, Relkin E, Slepecky NB, Sterns A, Warr WB, Weekly JM, Woods CI (1999) Olivocochlear neurons in the chinchilla: a retrograde fluorescent labelling study. Hear Res 134:57-70.

Carmel PW, Starr A (1964) Non-acoustic factors influencing activity of middle ear muscles in waking cats. Nature 202:195-196.

Chiba AA, Bushnell PJ, Oshiro WM, Gallagher M (1999) Selective removal of cholinergic neurons in the basal forebrain alters cued target detection. NeuroReport 10:3119-3123.

Cooper NP, Guinan Jr JJ (2003) Separate mechanical processes underlie fast and slow effects of medial olivocochlear efferent activity. J Physiol (Lond) 548:307-312.

Dai HP, Scharf B, Buus S (1991) Effective attenuation of signals in noise under focused attention. J Acoust Soc Am 89:2837-2842.

Dallos P (1992) The active cochlea. J Neurosci 12:4575-4585.

Dallos P, Cheatham MA (1976) Production of cochlear potentials by inner and outer hair cells. J Acoust Soc Am 60:510-512.

Dolan DF, Guo MH, Nuttall AL (1997) Frequency-dependent enhancement of basilar membrane velocity during olivocochlear bundle stimulation. J Acoust Soc Am 102:3587-3596.
Duncan J, Ward R, Shapiro K (1994) Direct measurement of attentional dwell time in human vision. Nature 369:313-315.

Dunlop CW, Webster WR, Simons LA (1965) Effect of attention on evoked responses in the classical auditory pathway. Nature 206:1048-1050.

Feliciano M, Saldaña E, Mugnaini E (1995) Direct projections from the rat primary auditory neocortex to nucleus sagulum, paralemniscal regions, superior olivary complex and cochlear nuclei. Aud Neurosci $1: 287-308$

Fex J (1959) Augmentation of cochlear microphonic by stimulation of efferent fibres to the cochlea; preliminary report. Acta Otolaryngol 50:540-541.

Galambos R (1956) Suppression of auditory nerve activity by stimulation of efferent fibers to cochlea. J Neurophysiol 19:424-437.

Gifford ML, Guinan Jr JJ (1987) Effects of electrical stimulation of medial olivocochlear neurons on ipsilateral and contralateral cochlear responses. Hear Res 29:179-194.

Greenberg GZ, Larkin WD (1968) Frequency-response characteristic of auditory observers detecting signals of a single frequency in noise: the probesignal method. J Acoust Soc Am 44:1513-1523.

Groff JA, Liberman MC (2003) Modulation of cochlear afferent response by the lateral olivocochlear system: activation via electrical stimulation of the inferior colliculus. J Neurophysiol 90:3178-3200.

Guinan Jr JJ (1996) Physiology of olivocochlear efferents. In: The cochlea (Dallos P, Popper A, Fay R, eds), pp 435-502. New York: Springer.

Hamame CM, Delano PH, Robles L (2006) Relevance of a neutral cue in a two choice detection task in the rat. Biol Res 39:259-267.

Hernandez-Peón R, Scherrer H, Jouvet M (1956) Modification of electric activity in cochlear nucleus during attention in unanesthetized cats. Science 123:331-332.

James AL, Harrison RV, Pienkowski M, Dajani HR, Mount RJ (2005) Dynamics of real time DPOAE contralateral suppression in chinchillas and humans. Int J Audiol 44:118-129.

Kawase T, Liberman MC (1993) Antimasking effects of the olivocochlear reflex. I. Enhancement of compound action potentials to masked tones. J Neurophysiol 70:2519-2532.

Lukas JH (1980) Human auditory attention: the olivocochlear bundle may function as a peripheral filter. Psychophysiology 17:444-452.

Maison SF, Liberman MC (2000) Predicting vulnerability to acoustic injury with a noninvasive assay of olivocochlear reflex strength. J Neurosci 20:4701-4707.

Muir JL, Everitt BJ, Robbins TW (1994) AMPA-induced excitotoxic lesions of the basal forebrain: a significant role for the cortical cholinergic system in attentional function. J Neurosci 14:2313-2326.

Mulders WH, Robertson D (2000) Evidence for direct cortical innervation of medial olivocochlear neurones in rats. Hear Res 144:65-72.

Muller MM, Teder-Salejarvi W, Hillyard SA (1998) The time course of cortical facilitation during cued shifts of spatial attention. Nat Neurosci 1:631-634.

Murugasu E, Russell IJ (1996) The effect of efferent stimulation on basilar membrane displacement in the basal turn of the guinea pig cochlea. J Neurosci 16:325-332.

Oatman LC (1971) Role of visual attention on auditory evoked potentials in unanesthetized cats. Exp Neurol 32:341-356.

Oatman LC (1976) Effects of visual attention on the intensity of auditory evoked potentials. Exp Neurol 51:41-53.

Oatman LC, Anderson BW (1977) Effects of visual attention on tone burst evoked auditory potentials. Exp Neurol 57:200-211.

Oliver D, Klocker N, Schuck J, Baukrowitz T, Ruppersberg JP, Fakler B (2000) Gating of $\mathrm{Ca}^{2+}$-activated $\mathrm{K}^{+}$channels controls fast inhibitory synaptic transmission at auditory outer hair cells. Neuron 26:595601.

Picton TW, Hillyard SA (1974) Human auditory evoked potentials. II. Effects of attention. Electroencephalogr Clin Neurophysiol 36:191-199.

Picton TW, Hillyard SA, Galambos R, Schiff M (1971) Human auditory attention: a central or peripheral process? Science 173:351-353.

Polley DB, Steinberg EE, Merzenich MM (2006) Perceptual learning directs auditory cortical map reorganization through top-down influences. J Neurosci 26:4970-4982.

Puel JL, Bonfils P, Pujol R (1988) Selective attention modifies the active micromechanical properties of the cochlea. Brain Res 447:380-383.

Puria S, Guinan Jr JJ, Liberman MC (1996) Olivocochlear reflex assays: ef- 
fects of contralateral sound on compound action potentials versus earcanal distortion products. J Acoust Soc Am 99:500-507.

Robles L, Ruggero MA (2001) Mechanics of the mammalian cochlea. Physiol Rev 81:1305-1352.

Romo R, Salinas E (2003) Flutter discrimination: neural codes, perception, memory and decision making. Nat Rev Neurosci 4:203-218.

Scharf B, Magnan J, Chays A (1997) On the role of the olivocochlear bundle in hearing: 16 case studies. Hear Res 103:101-122.

Starr A (1964) Influence of motor activity on click-evoked responses in the auditory pathway of waking cats. Exp Neurol 10:191-204.

Velluti R, Pedemonte M, Garcia-Austt E (1989) Correlative changes of auditory nerve and microphonic potentials throughout sleep. Hear Res 39:203-208.

Ward R, Duncan J, Shapiro K (1996) The slow time-course of visual attention. Cognit Psychol 30:79-109.
Warr WB (1975) Olivocochlear and vestibular efferent neurons of the feline brain stem: their location, morphology and number determined by retrograde axonal transport and acetylcholinesterase histochemistry. J Comp Neurol 161:159-181.

Wickelgren WO (1968) Effects of walking and flash stimulation on clickevoked responses in cats. J Neurophysiol 31:769-776.

Woldorff MG, Gallen CC, Hampson SA, Hillyard SA, Pantev C, Sobel D, Bloom FE (1993) Modulation of early sensory processing in human auditory cortex during auditory selective attention. Proc Natl Acad Sci USA 90:8722-8726.

Zheng XY, McFadden SL, Henderson D, Ding DL, Burkard R (2000) Cochlear microphonics and otoacoustic emissions in chronically deefferented chinchilla. Hear Res 143:14-22. 